\title{
The Searching Model and Strategy for Passive Sensor Cue of Phased Array Radar
}

\author{
TANG Shujuan ${ }^{a}$, BI Duyan, XU Yunshan, WANG Weijia \\ Institute of Aeronautics and Astronautics Engineering, Air Force Engineering University, 710038,Xi'an, China
}

\begin{abstract}
For the problem of passive sensor cueing of phase array radar for target searching, the static and dynamic cueing model is given on the basis of characteristics of cueing sensors. considered the precision difference of cueing passive sensors, a probability density model under the case of multiple targets, and a kind of effective search tactics that guide the arranged of beams position are studied and presented. The Effectiveness of the model is proved by analysis and comparision from the results obtained by simulation
\end{abstract}

\section{Introduction}

Various kinds of sensors have been centrally configured on airborne warning and detection system, including both passive sensors and active sensors. For getting full use of the performance-advantage of multikinds sensors, it is a significant way of sensorscooperation to achieve cooperating-detection by passive sensor cueing of phase array radar, especially in case of multi-targets situation and complex movement characteristics on battlefield, problems of phased array radar under the cueing information capture target rapidly and accurately is very critical and worth working.

Document [1,2] described sensor cueing method and factors that may influence the result of cueing, laid great foundation for sensor target cueing problems. Document [3-4] introduced the target cueing demand for modern fleet air defense combat and its influence on operational capability. Document [5] proposed a novel cued search stratege based on information gain for phased array radar. Document [6] made a further research of IRST guiding radar under multi-target circumstance, built sensor cueing model and executed simulation verification on condition that target location remained constant while observing. Document [7-8] analyzed the sensor cueing and handoff based on task driven and distributed search under airborn centralize sensors configuration circumstance. These methods above were not considered the precision difference of cueing passive sensors.

This article focused on passive sensor target cueing of phased array radar under single airborne platform multi-sensor face to multi-objective situation, establish static and dynamic cueing model on the basis of characteristics of cueing sensors, then analyze specific searching strategy and simulative verify the abovementioned searching strategy in typical environment.

\section{Target Cueing model}

Target hand-off from passive sensor to radar is a typical application of wide-filed sensor cueing of narrowfiled sensor. As is shown in Fig. $1, V_{l}$ is the viewing range of narrow-field sensor $S_{1}, V_{2}$ is the viewing range of wide-field sensor $S_{2}$, while executing target hand-off, $S_{I}$ need to immediately adjust its viewing direction and focus the viewing center to $\theta$ under the cueing information from $S_{2}$.

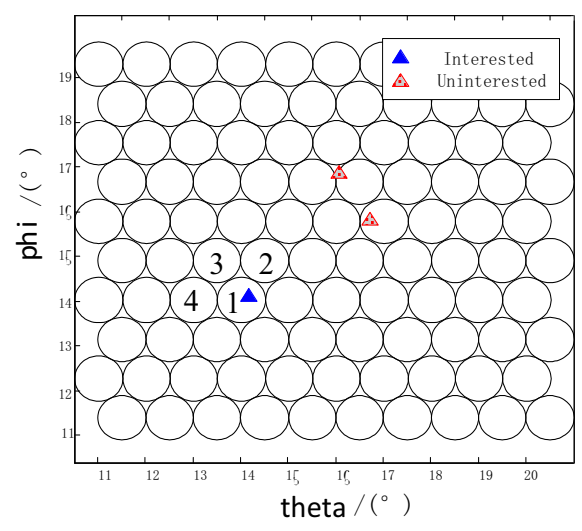

Figure.1.Cueing and hand-off diagram

\subsection{Static cueing model}

Hand-off process of static cueing, requires the measurement error of target cueing sensor is lesser than the viewing range of sensor being cued. Suppose $\theta$ is the real position of target, $\hat{\theta}$ is the estimated position of target from cueing information, if the cueing error

\footnotetext{
${ }^{\mathrm{a}}$ Corresponding author: TANG Shujuan. email:busybring@163.com
} 
$\varepsilon=\theta-\hat{\theta}$ drop in a range of one single radar beam, theoretically radar beam can directly intercept target while focus on $\hat{\theta}^{[9]}$.

However, due to the relative movement between sensor platform and target, $\hat{\theta}$ changes during the target hand-off process. Assume the maximum of cueing error caused by relative movement is $S_{\max }$, the real position of target can be defined as a random variable $\theta_{l}$ having the uniform distribution with the range [ $\theta-S_{\max }, \theta+\mathrm{S}_{\max }$ ], Due to the long distance between target and sensor platform and the little time of the cueing and hand-off process, cueing error caused by relative movement often remains minor, which could be ignore.

\subsection{Static cueing model}

Usually, it's difficult for some sensors to reach the certain cueing precision, thus possibility of target interception only occurs when radar executes searching in relative-large space under cueing information during target hand-off process, this way of cueing is called dynamic cueing.

Cueing information error not only contains system errors due to limitation of measuring and signal processing ability of sensor, but also contains random errors caused by other uncertain factor, thus the target cueing information provided by passive sensor are superposed by the two parts: target real position and cueing error.

$$
(\hat{\theta}, \hat{\varphi})=(\theta, \varphi)+\left(\varepsilon_{\theta}, \varepsilon_{\varphi}\right)
$$

Suppose $\left(\varepsilon_{\theta}, \varepsilon_{\varphi}\right)$ obey 2D joint Gaussian distribution and having independence in position and pitching direction, the probability density function of target dropping in one same area is expressed as:

$$
\mathrm{f}(\theta, \varphi)=\frac{1}{2 \pi \sigma_{\theta} \sigma_{\varphi}} \exp \left[-\frac{(\theta-\hat{\theta})^{2}}{2 \sigma_{\theta}^{2}}-\frac{(\varphi-\hat{\varphi})^{2}}{2 \sigma_{\kappa}^{2}}\right]
$$

To integrate the above-mentioned equation with respect of position and pitching direction, the probability of target dropping in area $S$ is obtained as:

$$
P_{\mathrm{c}}=\iint_{S} \mathrm{f}(\theta, \varphi) \mathrm{d} \theta d \varphi
$$

$P_{c}$ was defined as target drop-in interception in area $S$, it's the evidence to confirm searching range of sensor cued and generate searching strategy under dynamic cueing.

While cueing information error being large, specific target capturing requires enlargement of searching space, and then may possibly get influenced by other interrupting targets.

\section{Analysis of phase array radar searching Strategy}

\subsection{Beam patterns and scanning sequence}

Typical beam position arrangement patterns are shown in Fig2. Document [9] compared the parameters in beam coverage rate, coverage blind area and beam overlap rate. In practicality, beam position arrangement patterns maybe made certain or dynamic optimized. Considering the Gaussian distribution of the probability density function of target dropping in searching airspace, choose the corss beam pattern can brought high beam coverage rate while low coverage blind area and beam overlap rate.

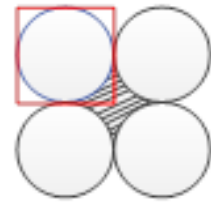

(a) column beams

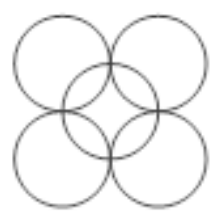

(c)superposition beams

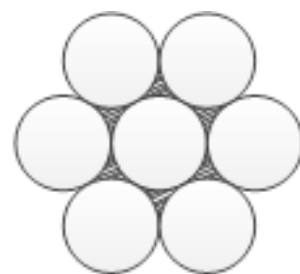

(b) corss beams

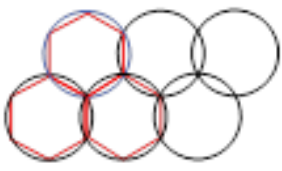

(d)honeycomb beam
Figure.2 Radar beam position arrangement patterns

In traditional mechanical scanning radar, the usual beam scanning sequences are raster scanning and sawtooth scaning ${ }^{[10]}$, as shown in Fig3. Based on excellent beam agile capability and effective resource management, phased array radars (PAR) have greater capabilities than mechanical scanning radar. Compare with the conventional method of sequential search, passive sensor cueing PAR for target searching improve the search performance and save radar time resources.

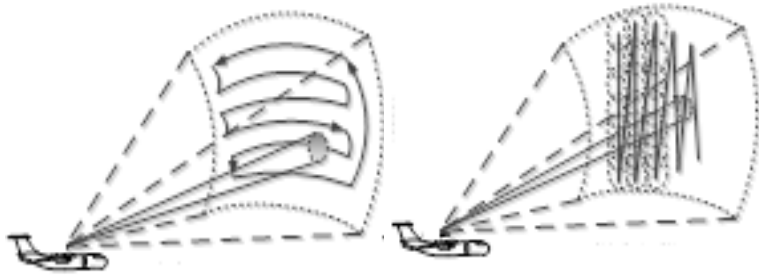

(a) raster scanning (b) sawtooth scaning

Figure.3. Beam scanning sequence

\subsection{Searching strategy}

Whether it is static or dynamic cueing model, a definite capture probability threshold $P_{0}$ is not the only required thing of radar searching, a time-sensitive parameter $T_{0}$ is also required. Under dynamic cueing 
model, false hand-off caused by interference from other targets is also worth considering.

Define searching space as the largest space can be searching of in one single hand-off process ${ }^{[11]}$, expressed as $\Omega$. Actually, successful probability of cueing is the appearance probability of target No.0 in phase array radar searching space $\Omega$. For convenience of description, simplify formula (3) as follows, $f_{0}(z)$ expresses the appearance probability of target No.0 in space $\Omega$ :

$$
P_{d}=\int_{\Omega} f_{0}(z) d z
$$

Define the maximum amount of searching beam position in limited time is $N_{0}$, corresponding searching space $\Omega$ is a set with cardinality of $\mathrm{N}_{0}$.

When cueing error is too large to ignore the interfering target in searching space, false hand-off probability of capturing the non-hand-off target(suppose is target No.0) is defined as $P_{f}$ :

$$
P_{f}=\int_{\Omega} \sum_{i=1}^{N} f_{i}(z) d z / \int_{\Omega} \sum_{k=0}^{N} f_{k}(z) d z
$$

While radar searching targets, false hand-off probability shall be lower than a certain threshold value defined as $\mathrm{P}_{f 0}$.

Due to the flexibility of phase array radar beam control, this article gives searching strategy as follow: When phase array radar is cued by passive sensor, the searching strategy is shown in formula (6)

$$
\begin{aligned}
& \Omega_{o p t}=\arg \max P_{d} \\
& \text { s.t. } \quad\left\{\begin{array}{c}
\int_{\Omega} f_{0}(z) d z \geq P_{0} \\
N(\Omega) \leq N_{0}, \Omega=\left\{\Omega_{j}\right\}_{j=1}^{N_{0}} \\
P_{f} \leq P_{f 0}
\end{array}\right.
\end{aligned}
$$

Firstly, calculates the maximum probability of target appearance beam position $N_{0}$ as the initial searching space. Secondly, calculate the false hand-off probability in searching space, if it's lower than threshold, put the higher priority to which having the higher capturing probability, and cease search when the threshold value of cueing success rate is satisfied. If false hand-off probability in searching space is higher than the threshold, beam position with the largest false hand-off probability will be abandoned and beam position with the smallest false hand-off probability and similar interception probability will be joined to form a brand new searching space. The probability of target appearance is satisfied the request of hand-off, or fail by overstepping timeliness.

\section{Simulation Verification}

According to cueing information, suppose there are 3 targets in cueing space named No.0, No.1 and No.2 target (No.0 is the hand-off target) respectively having the direction and pitching position $\left[14^{\circ}, 14^{\circ}\right],\left[17^{\circ}, 16^{\circ}\right]$ and $\left[16^{\circ}, 17^{\circ}\right]$. Choose the corss beams pattern and the width of radar beam is $1^{\circ}$, capturing probability threshold set to $85 \%$, false hand-off probability threshold $P_{f 0}$ set to $20 \%$, hand-off timeliness set to timing of 40 beam positions.

Scene 1: Cueing information error standard deviation is $0.5^{\circ}$ both on azimuth and pitching, all conform with static cueing model. Simulation result is shown in Fig. 4.

Scene 2: Cueing information error standard deviation is $1.5^{\circ}$ both on azimuth and pitching, all conform with dynamic cueing model. Simulation result is shown in Fig.5.

Scene 3: Cueing information error standard deviation is $2.5^{\circ}$ both on azimuth and pitching, all conform with dynamic cueing model. Simulation result is shown in Fig.6.

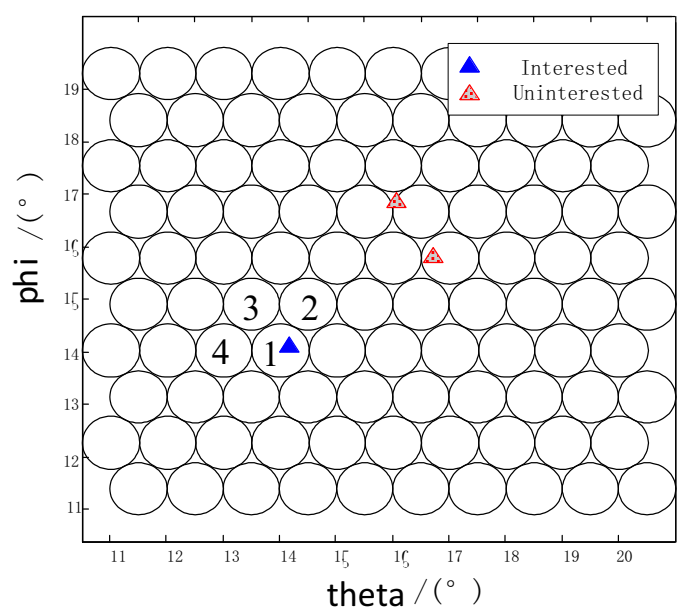

Figure.4 Searching Strategy of scene 1

As is shown in the figure 4 , beam position searching strategy is firstly point at the beam position of estimated cueing information (beam position 1 in figure 2). Because of the action of cueing information error, capturing probability on this beam position is only $46.6 \%$, which cannot reach capturing probability threshold and needs further enlargement of searching space. In sequence, search 4 beam positions which have high probability, capturing probability is obtained as $88.4 \%$, capturing probability threshold is reached, searching complete.

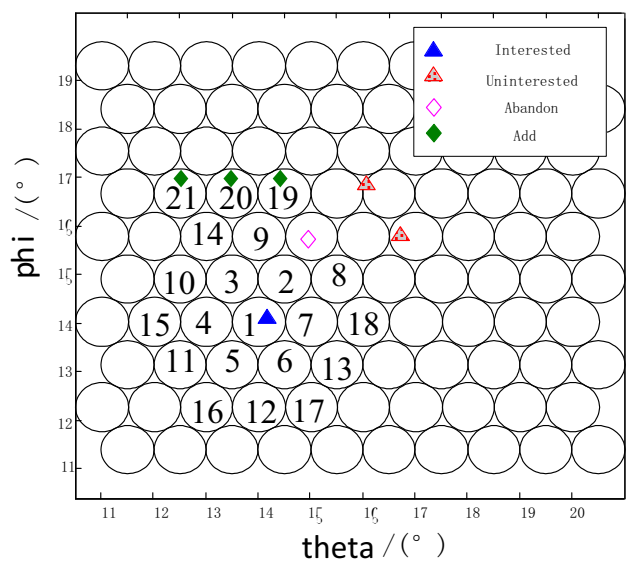

Figure.5. Searching Strategy of scene 2 
As is shown in the figure 5, beam position searching strategy is to firstly point at the beam position of estimated cueing information (beam position 1 in figure 3 ), searching space is larger than Scene 1 because of large cueing information error. While beam positions of the hollow diamond shown in the figure being searched, current beam position is abandoned for false hand-off probability being too large, beam positions of the solid diamond shown in the figure are chosen to continue the searching. When the eighteenth beam position is found, capturing probability threshold reached, searching finished. The final capturing probability is $85.4 \%$, false hand-off probability is $18.4 \%$.

From the comparison between scene 1 and scene 2, we can see that strategy from this article could well accomplish target capturing, increase veracity of target capturing by decreasing false hand-off probability when cueing error is fairly large.

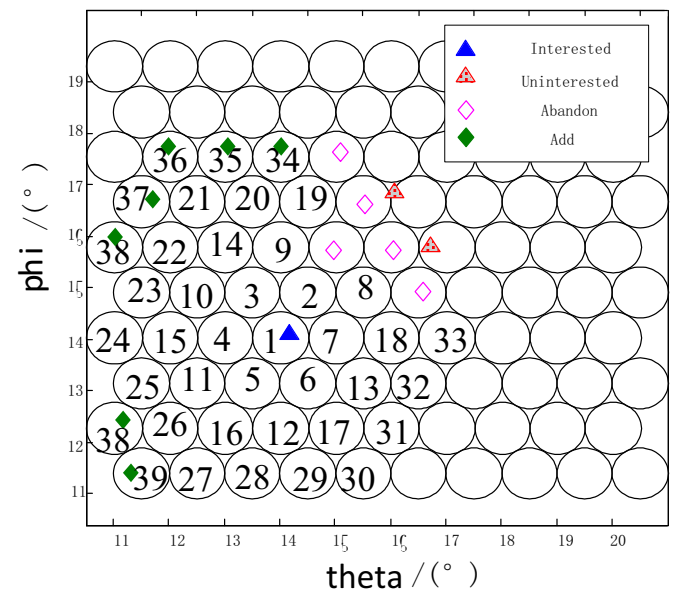

Figure.6 Searching Strategy of scene 1

Similarly, as is shown in the figure 6 , beam position is abandoned for false hand-off probability being too large, add beam positions are chosen to continue the searching. When the fortieth beam position is found, capturing probability cannot reach the probability threshold, hand-off failed, searching finished. The final capturing probability is $80.3 \%$, false hand-off probability is $14.8 \%$.

From the comparison between scene 2 and scene 3 , the increasing of cueing information error standard deviation lead to larger searching space, so searching times increased. If the last beam position is found but capturing probability cannot reach the probability threshold, hand-off failed.

For further analysis, the hand-off may fail by problem of large cueing error, which is mainly because of: low capturing probability due to dispersion of target appearance probability, overlarge false hand-off probability due to over-intensive target or timeliness being too strict etc. Therefore, in actual application, parameters such as hand-off timeliness $\mathrm{T}_{0}$, capturing probability threshold $\mathrm{P}_{0}$ and false hand-off probability threshold $\mathrm{P}_{f 0}$ shall be elected carefully according to real equipment and adjusted appropriately according to operational mission, in order to ensure the best result of cueing and hand-off.

\section{Conclusion}

While working, active sensor produces vast electromagnetic radiation, thus may reveal self-location and suffer interruption or attack from the hostile. Cooperation detection by using passive sensor cueing of active sensor is an efficient way to cope with invisible targets and electronic interference. This article has done a thorough research about the searching strategy of passive sensor cueing of phase array radar searching targets under multi-targets circumstance on space-based early warning detection platform with multi-sensors. Two kinds of target cueing models have been made according to precision difference of cueing information to analyze phase array radar searching strategy. Specific simulation verification of the above-mentioned searching strategy in typical environment are already executed. The result of this article is theoretically significant meaningful and have great engineering application value in centrally configured passive sensor cueing of active sensor cooperation detection.

\section{Acknowledgment}

We thank Yang Tao for their help of part of simulation analysis. This study was funded by the Aeronautical Science Foundation of China (No.20145596025)

\section{References}

1. Mahendra Mallick, Vikram Krishnamurthy Ba-Ngu Vo. Integrated Tracking, Classification, and Sensor Management[M]. Canada: The Institute of Electrical and Electronics Engineers, 2013.

2. Li Bi-cheng, Huang Jie, Gao Shi-hai, Wang Tian-peng. Information Fusion Technology and Application[M]. Beijing:National Defend Industy Press, 2010: 69-72.

3. Benaskeur A and Irandoust H.Sensor Management for Tactical Surveillance Operations[R].North Quebec City: Defence R\&D Canada, 2007:37-38.

4. Fan Hao, Huang Shucai, Gao Meifeng, WEI Daozhi. Research on Technique of Multi-Target Detection Using Multi-Sensor Cross-Cueing Based on Dynamic Coalition[J]. Journal of Astronautics, 2013(11): 2381-2386.

5. Lu Jianbin, Hu Weidong, Xiao Hui, etc. Novel cued search stratege based on information gain for phased array radar [J].Journal of Systems Engineering and Electronics, 2008, 19(2):292-297.

6. Zhang Hua-rui,Yang Hong-wen, Yu Wen-xian. The Handoff Method of IRST and Radar Under Multitarget Scenario[J]. Journal of Electronics \& Information Technology, 2011, 33(9): 19-24.

7. Zhao Jianheng, $\mathrm{Xu}$ Yunshan, Xiao Bingsong etc. Research on Sensor Indication and Handover Based 
on Task Driven[J] Computer Measurement \&Control. 2014. 22(7). 2277-2280

8. Yang Tao, Xu Yunshan, Tang Shujuan. Negotiation Mechanism of Sensor Indication and Handover Based on Distributed Search [J]. Fire Control \& Command Control . 2015. 40(1): 42:48

9. Li Zhi huai, Tan Xiansi, Wang Hong, etc. A model of the optimal beam position arrangement on tracking and guiding radar for near space hypersonic targets [J]. Journal of Optoelectronics. Laser. 2013. 24(4): 794-798

10. Ding Lufei, Geng Fulu, Cen Jianchun. Radar Principle[M]. Beijing. Publishing House of Electronics Industry. 2011

11. Lu Zhiwei, Li Ming, Ji Xiaoguang. Research on Radar searching Volume Based on Mutil-sensor Cooperation Technology[J]. Aeronautical Computing Technique, 2006, 36(5):28-31. 